EPJ Web of Conferences 88, 00018 (2015)

DOI: $10.1051 /$ epjconf/ 20158800018

(C) Owned by the authors, published by EDP Sciences - SIF, 2015

\title{
Constraining the nuclear matter equation of state around twice saturation density
}

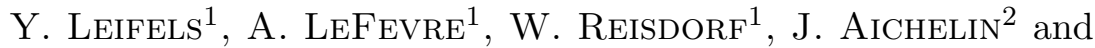 \\ C. HARTNACK ${ }^{2}$
}

${ }^{1}$ GSI Helmholtzzentrum für Schwerionenphysik GmbH, Planck Str. 1, D-64921 Darmstadt, Germany

${ }^{2}$ SUBATECH, University of Nantes - IN2P3/CNRS - Ecole des Mines de Nantes, 4 rue Alfred Kastler, F-44072 Nantes, France

\begin{abstract}
Using data on elliptic flow measured by the FOPI collaboration we extract constraints for the equation of state (EOS) of symmetric nuclear matter with the help of the microscopic transport code IQMD. Best agreement between data and calculations is obtained with a 'soft' equation of state including a momentum dependent interaction. From the model it can be deduced that the characteristic density related to the observed flow signal is around twice saturation density and that both compression within the fireball and the presence of the surrounding spectator matter is necessary for the development of the signal and its sensitivity to the nuclear equation of state.
\end{abstract}

\section{Introduction}

Understanding the equation of state (EOS) of nuclear matter is of fundamental importance in many areas of nuclear physics and astrophysics. The nuclear EOS describes the relation between density, pressure, energy, temperature and the asymmetry $\delta=\left(\rho_{n}-\rho_{p}\right) / \rho$, where $\rho_{n}, \rho_{n}$, and $\rho$ are neutron, proton and nuclear matter densities, respectively. It is an important ingredient in describing astrophysical objects like core collapse supernovae 
and compact stars. Calculation of the nuclear matter EOS from first principles is a complex task and the results need to be confronted to experimental data.

Heavy ion collisions provide the only tool to explore densities larger than the nuclear saturation density $\rho_{0}$ in the laboratory, as in the course of such collisions nuclear matter undergoes compression followed by expansion phases. In the incident energy regime between 0.4 and $2 \mathrm{~A} \mathrm{GeV}$ up to three times the nuclear saturation density is reached during the collisions. Several observables have been studied which are predicted to be sensitive to the nuclear EOS with the help of theoretical models: Kaon production in heavy ion reactions below threshold in NN collisions as measured by the KaoS collaboration [1] is sensitive to the density reached in the course of the reactions, which is depending on the stiffness of the equation of state. The authors claimed that only a soft EOS is consistent with the data, i.e. with a compression modulus at saturation density $\kappa \approx 200 \mathrm{MeV}$. This finding was confirmed by a second theoretical group and it was shown to be robust with respect to the input parameters to the models [2].

Flow phenomena were used to constrain the nuclear EOS in other investigations. By the EoS and the FOPI collaborations different flow observables were compared to the results of several microscopic transport codes [3,4]. But no stringent constraints on the nuclear EOS could be derived at that stage. In the study of Danielewicz et al. [5] side and elliptic flow data measured in $\mathrm{Au}+\mathrm{Au}$ collisions in the energy regime between 0.4 and $10 \mathrm{~A} \mathrm{GeV}$ have been compared to predictions of a BUU model. Again it was not possible to find a single parameterization for the nuclear EOS to describe consistently all experimental data which have been selected.

In order to improve this situation between 0.1 to $1.5 \mathrm{~A} \mathrm{GeV}$ bombarding energy we make use of extensive data on elliptic flow measured by the FOPI collaboration [6]. The data are compared to predictions of the Isospin Quantum Molecular Dynamics model using various phenomenological EOS [7]. Four Skyrme type parameterization are available in the code: H ('stiff'), S ('soft'), HM ('stiff momentum dependent'), SM ('soft momentum dependent'). Soft and hard EOS are characterised by the incompressibility at saturation density $K_{0}=200$ and $K_{0}=380$, respectively (for details see [7])

\section{Experimental data}

The collective flow phenomenon allows to reconstruct the reaction plane event-by-event and to study azimuthal correlations with respect to that 

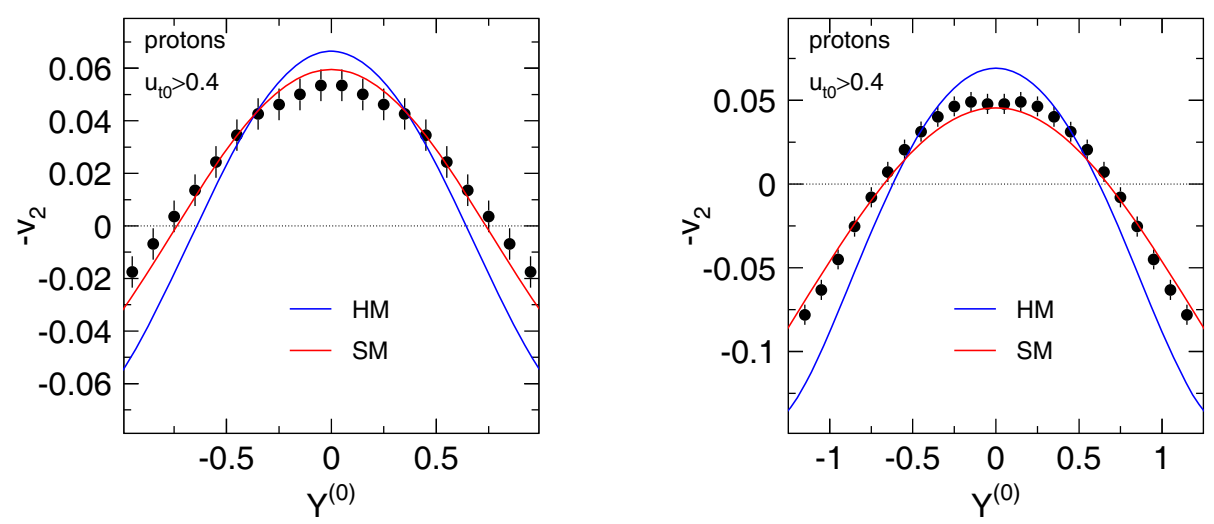

Figure 1: Left panel: Proton elliptic flow data, $-v_{2}\left(Y^{(0)}\right)$, in $\mathrm{Au}+\mathrm{Au}$ collisions at $0.4 \mathrm{~A} \mathrm{GeV}$ at $b_{0}<0.25$ together with IQMD-SM/HM simulations. Data and model results are from [6]. Right panel: Same for $1.5 \mathrm{~A} \mathrm{GeV}$.

plane. The resulting azimuthal distributions can be parameterized in terms of a Fourier expansion: $\frac{d N}{d y d \Phi}=v_{0}\left[1+2 v_{1} \cos (\Phi)+2 v_{2} \cos (2 \Phi)\right]$. The longitudinal (beam axis) rapidity $y$ is in the center of mass $(C M)$ reference system and the transverse (spatial) component $t$ of the four-velocity $u$ is given by $u_{t}=\beta_{t} \gamma$. The 3 -vector $\vec{\beta}$ is the velocity in units of the light velocity and $\gamma=1 / \sqrt{1-\beta^{2}}$. Scaled units $Y^{(0)}=y / y_{p}$ and $u_{t 0}=u_{t} / u_{p}$ are used, with $u_{p}=\beta_{p} \gamma_{p}$, the index $\mathrm{p}$ referring to the incident projectile in the $C M$. The transverse momentum method [5] was used including all particles detected outside the mid-rapidity interval $\left|Y^{(0)}\right|<0.3$ and excluding identified pions.

In Fig. 1 proton elliptic flow data $-v_{2}\left(Y^{(0)}\right)$ is shown for $0.4 \mathrm{~A} \mathrm{GeV}$ and $1.5 \mathrm{~A} \mathrm{GeV}$ and an impact parameter region $0.25<b / b_{\max }=b_{0}$, where $b_{\text {max }}=1.15\left(A_{P}^{1 / 3}+A_{T}^{1 / 3}\right)$ fm. Low transverse momenta $\left(u_{t 0}<0.4\right)$ have been cut due to apparatus limitations. The experimental data points (black dots with error bars) are shown together with results of IQMD calculations (HM blue and SM red). The experimental data is best described by a soft (SM) nuclear matter EOS. In former investigations only the mid-rapidity value of $v_{2}$ was compared to models, but obviously the whole shape $v_{2}\left(Y^{(0)}\right)$ is sensitive to the nuclear matter EOS. To take advantage of this fact the quantity $v_{2 n}=\left|v_{20}\right|+\left|v_{22}\right|$ is introduced where the parameters $v_{20}$ and $v_{22}$ are obtained by fitting to the elliptic flow data using $v_{2}\left(Y^{(0)}\right)=v_{20}+v_{22} \cdot Y^{(0)^{2}}$ in the scaled rapidity range $\left|Y^{(0)}\right|<0.8$. The results of this procedure are shown in Fig. 2. Experimental data points of $v_{2 n}$ for protons for $\mathrm{Au}+\mathrm{Au}$ collisions are shown as function of beam energy together with predictions of 


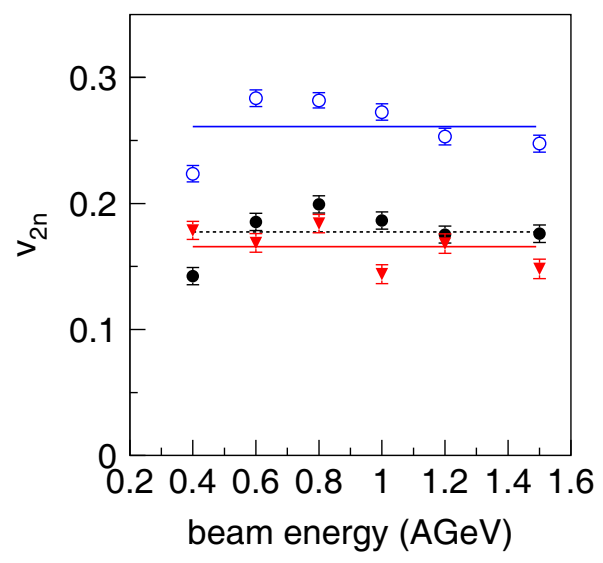

Figure 2: Observable $v_{2 n}$ for protons as a function of beam energy for $\mathrm{Au}+\mathrm{Au}$ collisions. Filled circles represent the experimental data, triangles depict IQMD results for SM and open circles for HM [6].

the IQMD model which have been treated in exactly the same manner as the experimental data. As the beam energy dependencies are rather weak, we indicate the average behaviour by straight lines. The comparison of the data for $v_{2 n}$ to calculations shows a preference for SM. The sensitivity is large: there is a factor $1.63 \pm 0.06$ between $\mathrm{HM}$ and $\mathrm{SM}$, a difference exceeding significantly the indicated experimental error bars.

\section{Model calculations}

The IQMD model describes not only the elliptic flow data measured for $\mathrm{Au}+\mathrm{Au}$ collisions in the energy regime between 0.4 and $1.5 \mathrm{~A} \mathrm{GeV}$ but also the Kaon data measured by the KaoS collaboration coming to the same conclusion with respect to the nuclear matter EOS. Hence, in two independent approaches a rather soft EOS $(\kappa \approx 200 \mathrm{MeV}$ was deduced. In addition, the model reproduces measured side flow data and pion production in the energy regime reasonably well by employing same SM-type equation of state. Hence, it is reasonable to assume that this model can also be used to investigate which times, densities and other conditions influence most the development of the elliptic flow.

In Fig. 3 the elliptic flow $v_{2}\left(Y^{(0)}\right)$ is plotted for different times during the collision. The times are scaled to the passing time $t / t_{\text {pass }}$, estimated from $t_{\text {pass }}=2 R / u_{p}$, where $u_{p}$ was defined in the previous section and $R$ is the radius of the colliding nuclei. The time zero corresponds to the instant 

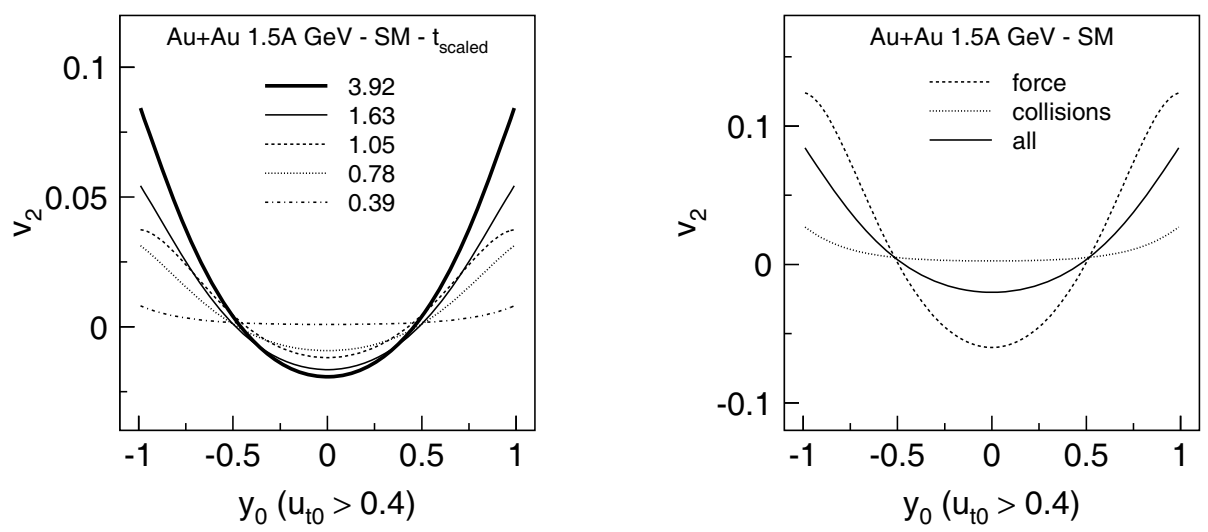

Figure 3: Left panel: Elliptic flow $v_{2}$ of protons having final transverse momenta $u_{t 0}>0.4$ as a function $Y^{(0)}$ at different $t / t_{\text {pass }}$. Right panel: Elliptic flow $v_{2}$ of protons with $u_{t 0}>0.4$ as a function of $Y^{(0)}$ for SM EOS at $t=60 \mathrm{fm} / \mathrm{c}$. The total signal ('all', full line) is shown along with its decompositions into the potential ('force', dashed line) and the collision part (dotted line).

when projectile and target nucleons have the first hard collision. Only protons are selected for this analysis which have finally transverse momenta $u_{t 0}>0.4$, which is the experimental cut adopted in the FOPI data. Such a selection removes most of the spectator particles.

One observes that the elliptic flow signal is developing fast at midrapidity where it is close to its final value already after the nuclei have passed each other, whereas $v_{2}$ at target and projectile rapidity stabilises only after two to three times of $t_{\text {pass }}$. The dependence is the same for SM and HM. The elliptic flow at target and projectile rapidities takes longer time to evolve since it originates also from late interactions with the spectator material. The difference between the the two extreme parameterizations of the nuclear EOS establishes already very early, i.e. when the squeeze-out signal is appearing. A decomposition of the elliptic flow in potential and collisional contributions elucidates the underlying reaction mechanisms. This is illustrated also in Fig. 3. In the right panel the final $v_{2}\left(Y^{(0)}\right)$ obtained by NN collisions is shown together with the contribution of the NN potential and the coherent sum of both. The IQMD models allows to record the momentum change due to collisions and potentials separately for each time step. Finally, integrating all contributions over the reaction time yields the total momentum transfer vector due to the potential and analogously by the collisions. Obviously, NN collisions do not contribute substantially to the 

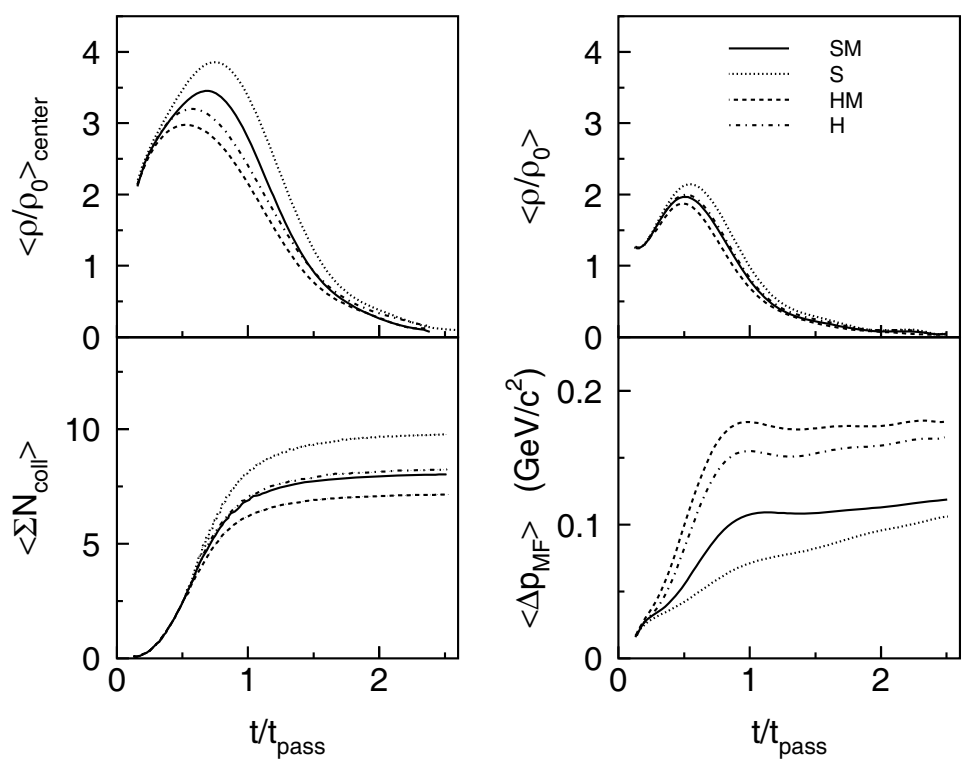

Figure 4: IQMD predictions of the time evolution of the proton characteristics in the $\mathrm{Au}+\mathrm{Au}$ collisions at $1.5 \mathrm{~A} \cdot \mathrm{GeV}$ incident energy and at an impact parameter $b=3 \mathrm{fm}$ for various parameterization of the EOS. The various quantities from left top to right bottom panels are: the reduced density probed by the protons at the center-of-mass of the colliding system, idem for all protons, the integrated (since time zero) sum of collisions performed by the protons, the integrated momentum change of protons due to the mean field (force).

elliptic flow signal, since the the resulting $v_{2}\left(Y^{(0)}\right)$ is essentially independent on $Y^{(0)}$. The potentials are mainly responsible for the observed signals instead.

This fact is further investigated in Fig. 4 where various quantities are plotted as a function of the scaled time $t / t_{\text {pass }}$ On the left hand side of the top panel the density, $\rho / \rho_{0}$ (normalised to the ground state density), is shown which is experienced by the protons in the central region of the collision for different versions of the EOS. The upper right panel depicts the mean density felt by all protons with transverse 4 -velocities $u_{t 0}>0.4$ in the final stage. The maximum density probed by these protons in the course of the collision is reached at around the full overlap of the incoming system $\left(t / t_{\text {pass }}=0.5\right)$. When employing a 'soft' EOS it takes a slightly longer time to reach the maximum of density and reaches higher densities as regard to the 'hard' EOS. The mean integrated number of collisions for all selected protons is shown in the lower left panel, and in the right panel the mean integrated momentum transfer due to potentials. Obviously until 
the passing time nearly all collisions are done, and most of the momentum transfer caused by the potential has been achieved, i.e. the influence of the potential becomes very small because the system is getting diluted. There is only a moderate dependence on the number of collisions when using a soft EOS instead of a hard one. However, the momentum transfer due to the potential in the 'hard' case is much larger than in the 'soft' one, which reflects of the higher repulsion of the hard equation of state. Similar conclusions can be drawn for all beam energies under consideration.

\section{Summary and conclusions}

A single parameter which is dubbed $v_{2 n}$ is characterising elliptic flow over a large rapidity interval ( $80 \%$ of the initial gap) and allows to discriminate rather clearly between a soft (SM) and a stiff (HM) EOS. $v_{2 n}$ differs by a factor 1.63 over an energy range extending from $0.4 A \mathrm{GeV}$ to $1.5 A \mathrm{GeV}$ when using IQMD, while the experiment allows to constrain $v_{2 n}$ within roughly a factor 1.1. The relevant density range can be estimated from IQMD simulations which reproduce the available experimental data in the beam energy regime under consideration reasonably well. This makes the 'flow method' competitive and complementary to the 'kaon method': the latter reaches such a high $\mathrm{SM} / \mathrm{HM}$ discrimination only around $0.8 A \mathrm{GeV}$ far below threshold. Within the IQMD model both methods yield the same results.

Acknowledgements: This work was supported by the Helmholtz International Center for FAIR within the framework of the LOEWE program (Landesoffensive zur Entwicklung Wissenschaftlich-Ökonomischer Exzellenz) launched by the State of Hesse and under the contract number 13-70 by the German-French Exchange Program supported by GSI and IN2P3.

\section{References}

[1] C. Sturm et al. [KAOS Collaboration], Phys. Rev. Lett. 86, 39 (2001)

[2] C. Hartnack et al., Phys. Rev. Lett. 96, 012302 (2006)

[3] S. Wang et al., Phys. Rev. Lett. 76, 3911 (1996).

[4] A. Andronic et al. [FOPI Collaboration], Phys. Lett. B 612, 173 (2005)

[5] P. Danielewicz et al., Science 298, 1592 (2002) 
EPJ Web of Conferences

[6] W. Reisdorf et al. [FOPI collaboration], Nucl. Phys. A 876 (2012) 1

[7] C. Hartnack et al., Eur. Phys. J. A 1 (1998) 151. 